\title{
Using Micro-Computed Tomography In Biomechanical Studies Of Rodent Bone
}

\author{
Sasidhar Uppuganti ${ }^{1}$ and Jeffry S. Nyman ${ }^{1,2}$ \\ ${ }^{1}$ Department of Orthopaedic Surgery and Rehabilitation/Vanderbilt University, Nashville, TN USA. \\ 2. Department of Veterans Affairs/ Tennessee Valley Healthcare System, Nashville, TN, USA
}

Pre-clinical studies involving experimental effects on rodent bones routinely include both microcomputed tomography $(\mu \mathrm{CT})$ analysis and biomechanical testing ex vivo. Being non-destructive, $\mu \mathrm{CT}$ analysis is done first to quantify structural and architectural parameters as well as mineral density. The whole bones are then typically subjected to bending or compression in a load-to-failure manner in order to quantify the biomechanical properties (Fig. 1). Combining $\mu$ CT-derived properties with the biomechanical properties provides estimates of material properties related to the inherent quality of the bone tissue. Such combinatorial analyses allow investigators to understand how an experimental manipulation affects the fracture resistance of bone. As a tutorial with emphasis on $\mu \mathrm{CT}$ methods, descriptions of two different rodent studies combining $\mu \mathrm{CT}$ with biomechanics follow.

As an example of how to determine whether a manipulation is affecting structural or material properties, we analyzed bones from mice lacking a matrix metalloproteinase (MMPs). Wild-type (WT), MMP-2 deficient $\left(\mathrm{Mmp}^{-/-}\right)$, and MMP-9 deficient mice $\left(\mathrm{Mmp}^{-{ }^{--}}\right)$on an FVB background (n=10/genotype) were euthanized at 16 weeks of age following an approved IACUC protocol [1]. Cleaned of soft tissue, the left femur was stored in saline (PBS) at $-20{ }^{\circ} \mathrm{C}$ until analyzed. The diaphysis of each femur $(2 \mathrm{~mm}$ centered at the mid-point) was scanned (Fig. 1) in a $\mu$ CT40 scanner (Scanco Medical; Switzerland) at an isotropic voxel size of $12 \mu \mathrm{m}$ to determine the average moment of inertia $\left(\mathrm{I}_{\mathrm{a}-\mathrm{p}}\right)$ per half of anteriorposterior width $\left(\mathrm{c}_{\mathrm{a}-\mathrm{p}}\right)$ and cross-sectional area (Ct.Ar). The femurs were monotonically loaded to failure in three-point bending (using material testing system) with the anterior surface down, a $7 \mathrm{~mm}$ span, and $3 \mathrm{~mm} / \mathrm{min}$ loading rate. Biomechanical properties were determined from the resulting forcedisplacement curve (Fig. 1). The estimated material properties were yield strength (flexural equation using yield force and $\mathrm{I}_{\mathrm{a}-\mathrm{p}} / \mathrm{c}_{\mathrm{a}-\mathrm{p}}$ ) and toughness (area-under-curve per Ct.Ar). The loss of the MMPs had differential effects on fracture resistance (Fig. 2).

Finite element analysis (FEA) is increasingly being used to assess the strength of bone because CT scans can readily be converted to finite element (FE) models. As an example of this technique, we analyzed mouse vertebral bodies (VBs) from a drug study. We treated 13-wk, male mice (FVB strain) with a control antibody $(13 \mathrm{C} 4, \mathrm{n}=7)$ or an anti-transforming growth factor beta (TGF- $\beta$ ) antibody (1D11, $n=8)$ for 4 weeks at the same dose $(10 \mathrm{mg} / \mathrm{kg} 3 \mathrm{x}$ per wk) [2]. After euthanasia, L6 VBs were harvested and stored frozen at $-20{ }^{\circ} \mathrm{C}$ in PBS. All VBs were scanned at an isotropic voxel size of $12 \mu \mathrm{m}$ using the same settings ( $\mu \mathrm{CT} 40$, Scanco Medical) and hydroxyapatite (HA) phantom calibration. To calculate BV/TV, contours were manually drawn for the region between the end plates (Fig. 3), whereas to generate the FE model, a circle with a constant radius was copied into each image between the end plates and positioned to transect the transverse processes, which do not bear load in the compression test (Fig. 3). A threshold of $421.4 \mathrm{mgHA} / \mathrm{cm}^{3}$ segmented bone from soft tissue and air. Then, using the Scanco elastic solver (8node brick elements), the equivalent strains were determined for high-friction, axial compression loading of each VB at 1\% apparent strain, elastic modulus (E) of $18 \mathrm{GPa}$, and Poisson's ratio of 0.03 for all elements (Fig. 3). The reaction force was determined for failure criteria in which $2 \%$ of the model volume exceeds $0.7 \%$ equivalent strain [3]. Each hydrated VB was subjected to axial compression to 
failure at $3 \mathrm{~mm} / \mathrm{min}$ in which the supporting platen had a rough surface and a moment relief to minimize slippage and off-axis loading. TGF- $\beta$ suppression by 1D11 increased the VB strength (Fig. 3)

\section{References:}

[1] Nyman, Lynch, Perrien et al, Journal of Bone and Mineral Research 26 (2011) p. 1252-60.

[2] Nyman et al, Orthopaedic Research Society (2013) p. 1.

[3] Pistoia, Van Rietbergen, Lockmuller et al, Bone 30 (2002) p. 842-48.

[4] The authors acknowledge research support from the DOD CDMRP, NSF, and NIH.
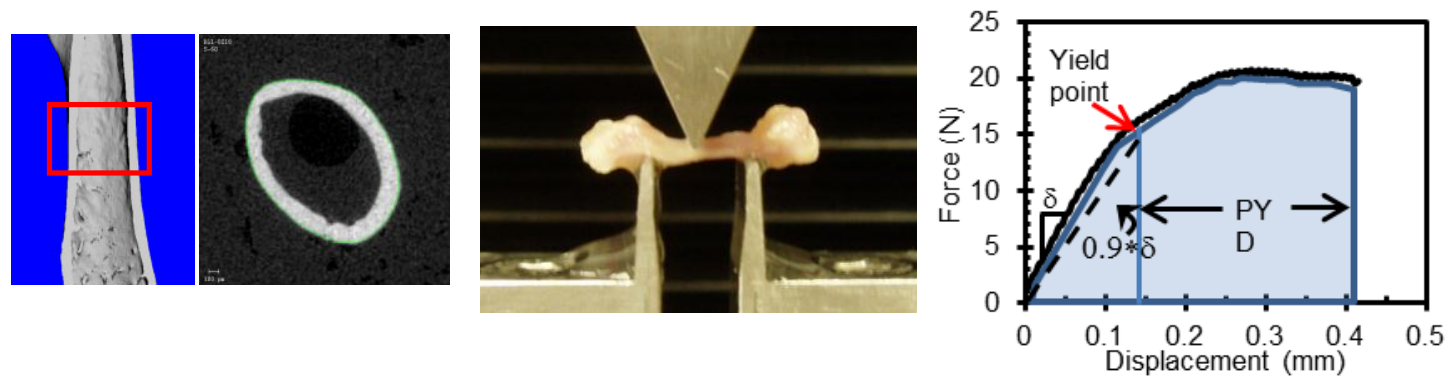

Figure 1. (Left to right) $3 \mathrm{D}$ rendered cut through image of a mouse femur diaphysis; $12 \mu \mathrm{m}$ transverse section of diaphysis within the red box; Three-point bending test of a hydrated mouse femur; Resulting force vs. displacement curve from which the biomechanical properties are determined.
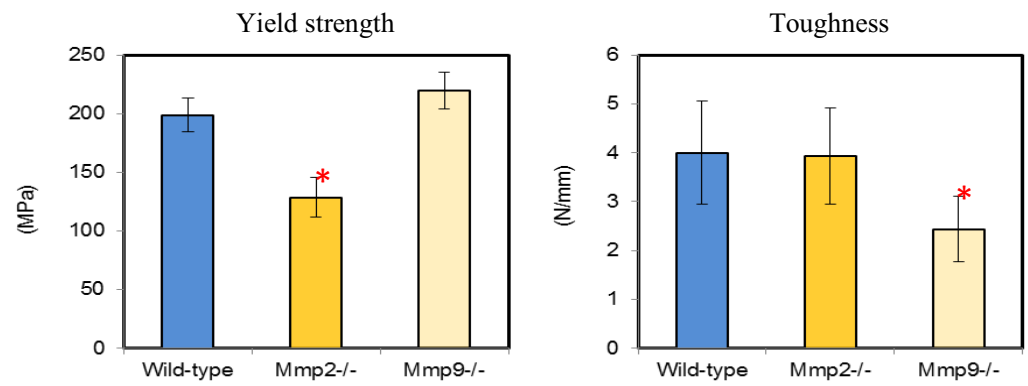

Figure 2. (Left) Comparison of yield strength (in MPa) between wild-type and MMP genotypes. (Right) Comparison of toughness (in N/mm) among wild-type and MMP phenotypes.
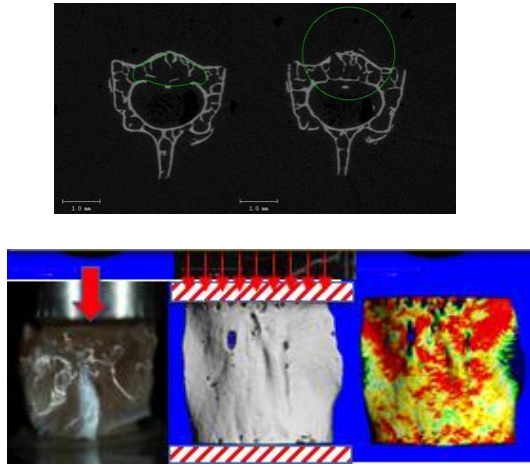

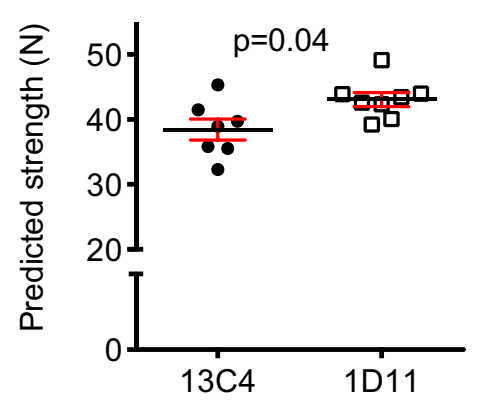

$18 \mathrm{GPa}, 2 \%, 0.7 \%, 450.8 \mathrm{mgHA} / \mathrm{cm}^{3}$

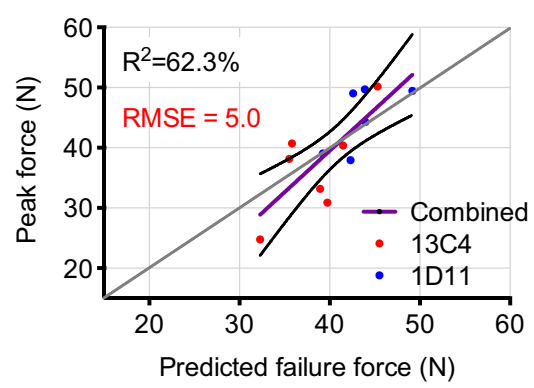

Figure 3. (Left to right) Transverse section contour for evaluating BV/TV and FE modeling (Top); Schematic of an experimental compression set-up and Scanco generated FEA (Bottom); VBs from the 1D11-treated mice were stronger; Experimental strength correlated with predicted strength. 